\title{
Dynamical Clustering Cooperative Spectrum Sensing with Bandwidth Constraints in CR Systems
}

\author{
Bing Wang ${ }^{1,2}$, Zhiquan Bai ${ }^{*}$, Yinlong $\mathrm{Xu}^{1}$, Peihao Dong ${ }^{1}$, and Kyungsup Kwak ${ }^{3}$ \\ ${ }^{1}$ School of Information Science and Engineering, Shandong University, China \\ ${ }^{2}$ Key Lab. of Higher Education of Sichuan Province for Enterprise Informationlization and Internet of Things, Sichuan \\ University of Science and Engineering, China \\ ${ }^{3}$ Graduate School of Information Technology and Telecommunications, INHA University, Korea \\ *Corresponding author: zqbai@sdu.edu.cn
}

\begin{abstract}
Cooperative spectrum sensing (CSS) scheme is employed to detect the primary user (PU) more quickly and accurately in cognitive radio (CR) systems. The performance of CSS scheme is usually significantly degraded when the inter-user and reporting channels experience path loss or deep fading. Meanwhile, the reporting channels are usually bandwidth limited. When the number of the secondary users (SUs) is very large, the sensing results need enormous bandwidth in the transmission. To solve this problem, a dynamical clustering CSS scheme with bandwidth constraints is proposed, where the SUs with better reporting channels are chosen as the cluster heads and the other SUs are dynamically grouped according to the inter-user channel conditions. In each cluster, the proposed scheme adopts the double thresholds scheme to make sure that only the SUs with high quality can send their sensing results. Simulation results demonstrate that the average number of sensing bits of the proposed scheme decreases greatly with a little loss in the sensing performance. This advantage is more obvious compared with the STBC based cooperative spectrum sensing scheme.
\end{abstract}

Keywords - Cognitive radio, cooperative spectrum sensing, dynamical clustering, double thresholds, bandwidth constraints

\section{INTRODUCTION}

Recently, great progress and remarkable development of wireless communication technologies have been made. With the emergence of new wireless applications, the competition for unlicensed radio spectrum becomes more and more intense. The spectrum demand of the unlicensed users (secondary users, SUs) is extremely great. However, according to the studies on the utilization of the radio spectrum, a large portion of the spectrum that is allocated to the licensed users (primary users, PUs) is actually idle at most time. The temporal and geographical utilization of the licensed spectrum only ranges from $15 \%$ to $85 \%$ [1]. As a desired solution to the dilemma of the spectrum scarcity and spectrum underutilization, cognitive radio (CR) technology proposed by Mitola [2] has been widely studied to improve the utilization efficiency of the radio spectrum.

Spectrum sensing (SS) is the key technology in CR system to improve the efficient utilization of spectrum by allowing SUs to use the "white space" [3] of licensed spectrum without causing harmful interference to the PUs. Usually, spectrum sensing of a single SU in the CR system bases on energy detection. However, when the detecting channels experience severe path loss or shadowing effect, the sensing performance of the SU may be degraded seriously and the hidden terminal problem may occur [4].

In order to improve the sensing performance, cooperative spectrum sensing (CSS) has been proposed [5][6]. Every single SU performs local energy detection independently and transmits the decision results to the fusion centre via the reporting channel. Then, the fusion centre will make a final judgement whether the PU exists or not. Given that the reporting channels are usually subject to shadowing and fading in practice, [7] proposes a cluster-based spectrum sensing scheme to improve the sensing performance, where the SUs are grouped into some clusters and the SU with the best reporting channel is chosen as the cluster head to transmit the detection results to the fusion centre. However, the above inter-user channels are considered to be perfect. Considering that all the wireless channels may experience the fading, a dynamic multi-user selection based CSS scheme is given in [8], which dynamically selects the best SUs as cluster heads and considers the fading effects of the inter-user channels. A CSS scheme with space-time block coding (STBC) is introduced in [4] to further improve the sensing performance.

However, the bandwidth needed in the transmission is usually limited [5]. If the number of SUs is very large, the bandwidth to transmit the detecting results may tend to be enormous. Double thresholds method is adopted in [9] to allow the SUs with enough information to report the sensing results to the cluster head merely. [10] has provided a further research in multi-cluster case. But both [9] and [10] assume that the inter-user channels and the reporting channels are perfect. In this paper, considering the practical imperfect channel conditions, we propose a dynamical clustering CSS scheme. SUs are dynamically grouped into clusters with a head user and the double thresholds method is used in each cluster. As a result, we can conclude that the proposed scheme could reduce the demand of bandwidth significantly with marginal loss in the sensing performance. This advantage is especially obvious compared with the STBC based CSS scheme.

The rest of the paper is organized as follows. In section II, the CSS model in CR system is described briefly. Section III explains the dynamical clustering cooperative spectrum sensing scheme with bandwidth constraints in detail. The 
simulation results and analysis are presented in section IV. Finally, we draw the conclusions in section V.

\section{Cooperative Spectrum SENSING IN CR SySTEM}

A practical CR system usually comprises primary users, secondary users, fusion centre and wireless channels. The wireless channels, including the detecting, reporting and interuser channels, usually experience fading or shadowing effects. The paper is mainly based on the assumption of Rayleigh fading channels which match well with the real wireless communications environments.

\section{A. Local Spectrum Sensing of a Single Secondary User}

In the practical CR systems, the SUs usually have no prior knowledge of the PU signals. Energy detection is an efficient method to detect the unknown signals [11]. Without loss of generality, the local spectrum sensing of a single secondary user is based on the energy detection in this paper.

According to the work of Harry Urkowitz about the energy detection theory [11], the detection probability $P_{d}$, the false alarm probability $P_{f}$, and the missing detection probability $P_{m}$ in the perfect environment are obtained respectively as,

$$
\begin{gathered}
P_{d}=Q_{m}(\sqrt{2 \gamma}, \sqrt{\lambda}), \\
P_{f}=\frac{\Gamma(m, \lambda / 2)}{\Gamma(m)}, \\
P_{m}=1-P_{d},
\end{gathered}
$$

where $\lambda$ is the decision threshold and $m=T W$ is the timebandwidth product. $\gamma$ is the signal-to-noise ratio (SNR) of the detecting channel. $Q_{m}(a, b)$ is the generalized Marcrum $Q$ function [12]. $\Gamma(a)$ and $\Gamma(a, b)$ are the complete and incomplete gamma function, respectively [13].

When the detecting channels experience Rayleigh fading, the average detection probability $P_{d}$ of the single SU can be given by [14],

$$
\begin{aligned}
P_{d} & =\int_{0}^{\infty} Q_{m}(\sqrt{2 \gamma}, \sqrt{\lambda}) f_{\gamma}(\gamma) d \gamma \\
& =\left(\frac{1+\bar{\gamma}}{\bar{\gamma}}\right)^{m-1} \times\left[e^{-\frac{\lambda}{2(1+\bar{\gamma})}}-e^{\left.-\frac{\lambda}{2} \sum_{k=0}^{m-2} \frac{1}{k !}\left(\frac{\lambda \bar{\gamma}}{2(1+\bar{\gamma})}\right)^{k}\right],}\right. \\
& +e^{-\frac{\lambda}{2}} \sum_{k=0}^{m-2} \frac{1}{k !}\left(\frac{\lambda}{2}\right)^{k}
\end{aligned}
$$

where $f_{\gamma}(x)$ denotes the probability density function (PDF) of SNR in Rayleigh fading channels. $\bar{\gamma}$ represents the average SNR of the Rayleigh fading channel. The instantaneous SNR of the Rayleigh fading channel follows an exponential distribution as,

$$
f_{\gamma}(\gamma)=\frac{1}{\bar{\gamma}} e^{\frac{\gamma}{\bar{\gamma}}}, \quad \gamma \geq 0 .
$$

Meanwhile, $P_{f}$ is independent of the SNR of the detecting channel and remains the same as formula (2).

\section{B. Cooperative Spectrum Sensing}

When the detecting channels experience severe fading or shadowing effects, the hidden terminal problem may occur. To solve the problem and improve the sensing performance, CSS is proposed, where each SU performs local energy detection and then transmits a 1-bit decision to the fusion centre which adopts or-rule to make the final decision.

According to [7], the probabilities of detection, false alarm and missing detection of CSS scheme under Rayleigh fading conditions are given by the following formulas, respectively,

$$
\begin{gathered}
Q_{d}=1-\prod_{i=1}^{N}\left[P_{d, i} P_{e, i}+\left(1-P_{d, i}\right)\left(1-P_{e, i}\right)\right], \\
Q_{f}=1-\prod_{i=1}^{N}\left[P_{f, i} P_{e, i}+\left(1-P_{f, i}\right)\left(1-P_{e, i}\right)\right], \\
Q_{m}=\prod_{i=1}^{N}\left[P_{m, i}\left(1-P_{e, i}\right)+\left(1-P_{m, i}\right) P_{e, i}\right],
\end{gathered}
$$

where $N$ is the number of the SUs, $P_{d, i}, P_{f, i}$ and $P_{m, i}$ are the $i$ th SU's local probabilities of detection, false alarm and missing detection, respectively. $P_{e, i}$ represents the bit error probability (BEP) of the Rayleigh fading reporting channel between the fusion centre and the $i$ th SU. When the channel transmits BPSK signals, $P_{e, i}$ can be calculated as [8],

$$
P_{e, i}=\frac{1}{2}\left(1-\sqrt{\frac{\bar{\rho}}{1+\bar{\rho}}}\right),
$$

where $\bar{\rho}$ denotes the average SNR of the reporting channel.

The sensing performance of conventional CSS will degrade greatly if the reporting channels subject to serious fading. STBC technology can be applied in the CSS to decrease the $\mathrm{BEP}$ of the reporting channels and reduce the impacts on the sensing performance caused by the reporting channels.

\section{Dynamical Clustering CoOperative SPECTRUM SENSING SCHEME WITH BANDWIDTH CONSTRAINTS}

\section{A. Dynamical Clustering Method}

Considering all the channels experience fading, the sensing performance may be weakened if the fading of the inter-user channels is disregarded in grouping the SUs. To alleviate the impact of the fading in inter-user channels, a dynamical clustering process is performed as follows.

Firstly, the SU with the largest SNR is selected as a cluster head, and then the potential SU and the head user form a network with the fusion centre to judge whether the potential SU should join the head SU or not, as shown in Figure 1.

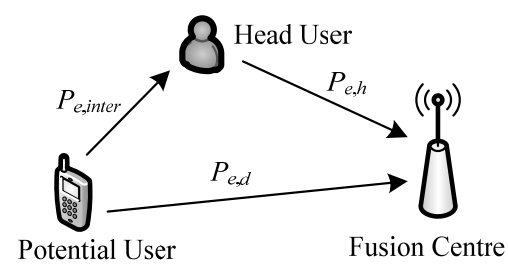

Figure 1. Relationship of potential user, head user and fusion centre

$P_{e, d}$ and $P_{e, h}$ represent the BEPs when the potential user and the head user directly send message to the fusion centre. If the 
potential user first sends its message to the head user and then the head user transfers the message to the fusion centre, the BEP $P_{e, t}$ can be obtained as,

$$
P_{e, t}=1-\left(1-P_{e, \text { inter }}\right)\left(1-P_{e, h}\right)-P_{e, \text { inter }} P_{e, h} .
$$

If $P_{e, t} \leq P_{e, d}$, the potential user joins the head user as its member, if not, the potential user does not joint the head user.

Repeat the above procedures among the left SUs until all the SUs are grouped. The clustering process is over.

\section{B. Double Thresholds Method}

In the CR systems, the sensing results are transmitted through a control channel which is usually bandwidth constrained. When the number of the SUs is large, the necessary communication bandwidth can be enormous.

After the clustering process, two thresholds, $\lambda_{1}$ and $\lambda_{2}$, are used to justify the reliability of the SU. As shown in Figure 2, the energy statistic $E$ of a single SU can be located in three assumption areas.

Let $H_{1}$ denote that the primary user exists and $H_{0}$ represent the primary user is absent. If the collected energy $E$ of a single SU exceeds the threshold $\lambda_{2}$, it will make decision $H_{1}$. If energy $E$ is below the threshold $\lambda_{1}$, decision $H_{0}$ will be made. Otherwise, the SUs do not make any decision. The SUs with decisions are considered to be reliable and allowed to report their local binary decision results.

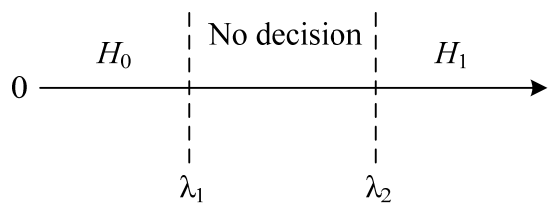

Figure 2. Assumption areas with double thresholds

1) Sensing Bits Analysis: The dynamical clustering CSS with bandwidth constraint is demonstrated in Figure 3. All the SUs are formed into clusters and only the SUs with reliable sensing information report the results to the head SU in each cluster. Then, the head SUs send the collected results to the fusion centre.

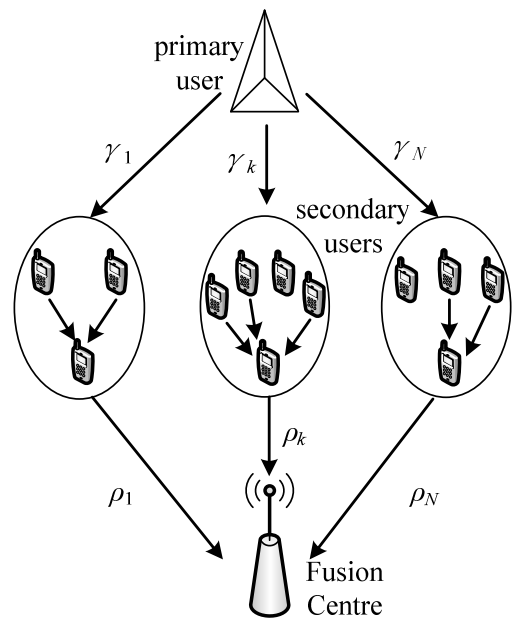

Figure 3. Dynamical clustering cooperative sepctrum sensing with bandwidth constraints
Assume that there are $N$ clusters and the number of the SUs in the $i$ th cluster is $N_{i}$. Without loss of generality, we take the $i$ th cluster for an elaborate analysis.

Let $L$ denote the SU making decision during the sensing and $L$ ' denote the opposite event. $E_{i, j}$ represents the energy statistic of the $j$ th SU in the $i$ th cluster. $\lambda_{1, i}$ and $\lambda_{2, i}$ are the low and high thresholds of the $i$ th cluster, respectively.

The probabilities of the two events can be obtained as,

$$
\begin{gathered}
P\left\{L^{\prime}\right\}=P\left\{\lambda_{1, i}<E_{i, j}<\lambda_{2, i}\right\}, \\
P\{L\}=1-P\left\{\lambda_{1, i}<E_{i, j}<\lambda_{2, i}\right\}=1-P\left\{L^{\prime}\right\},
\end{gathered}
$$

If $P_{0, i}$ and $P_{1, i}$ represent the probabilities of the absence and existence of the PU in the $i$ th cluster, the number of the sensing bits in the $i$ th cluster can be given as,

$$
\begin{aligned}
n_{i} & =P_{0, i} \sum_{k=1}^{N_{i}} k\left(\begin{array}{c}
N_{i} \\
k
\end{array}\right)\left[P\left\{L \mid H_{0}\right\}\right]^{k}\left[P\left\{L^{\prime} \mid H_{0}\right\}\right]^{N_{i}-k} \\
& +P_{1, i} \sum_{k=1}^{N_{i}} k\left(\begin{array}{c}
N_{i} \\
k
\end{array}\right)\left[P\left\{L \mid H_{1}\right\}\right]^{k}\left[P\left\{L^{\prime} \mid H_{1}\right\}\right]^{N_{i}-k},
\end{aligned}
$$

Consequently, we have

$$
\begin{aligned}
n_{i} & =P_{0, i} \cdot N_{i} \cdot\left(1-P\left\{\lambda_{1, i}<E_{i, j}<\lambda_{2, i} \mid H_{0}\right\}\right) \\
& +P_{1, i} \cdot N_{i} \cdot\left(1-P\left\{\lambda_{1, i}<E_{i, j}<\lambda_{2, i} \mid H_{1}\right\}\right) \\
& =N_{i}\left(P_{0, i}+P_{1, i}\right)-N_{i} P_{0, i} \Delta_{0, i}-N_{i} P_{1, i} \Delta_{1, i} \\
& =N_{i}\left(1-P_{0, i} \Delta_{0, i}-P_{1, i} \Delta_{1, i}\right)
\end{aligned}
$$

where $\Delta_{0, i}=P\left\{\lambda_{1, i}<E_{i, j}<\lambda_{2, i} \mid H_{0}\right\}$ and $\Delta_{1, i}=P\left\{\lambda_{1, i}<E_{i, j}<\lambda_{2, i} \mid H_{1}\right\}$ are the probabilities of making no decisions for the $j$ th SU in the $i$ th cluster under the assumptions $H_{0}$ and $H_{1}$, respectively.

From (14), we find that $n_{i}$ is smaller than $N_{i}$. Hence, the proposed scheme can save the bandwidth compared with the other schemes.

2) Sensing Performance Analysis: Different from [9] and [10], we assume that all the channels experience Rayleigh fading and consider the influence of the inter-user channels.

Let $F(\lambda)$ and $G(\lambda)$ denote the cumulative probability function (CPF) of the energy statistic $E$ of the single SU under the hypothesis $H_{0}$ and $H_{1}$, respectively. Then we have [14]

$$
\begin{gathered}
F(\lambda)=\int_{0}^{\lambda} f\left(E \mid H_{0}\right) d E=1-P_{f}=1-\frac{\Gamma(m, \lambda / 2)}{\Gamma(m)} \\
G(\lambda)=\int_{0}^{\lambda} f\left(E \mid H_{1}\right) d E=1-P_{d}=1-e^{-\frac{\lambda}{2}} \sum_{k=0}^{m-2} \frac{1}{k !}\left(\frac{\lambda}{2}\right)^{k} \\
-\left(\frac{1+\bar{\gamma}}{\bar{\gamma}}\right)^{m-1}\left[e^{-\frac{\lambda}{2(1+\bar{\gamma})}}-e^{-\frac{\lambda}{2}} \sum_{k=0}^{m-2} \frac{1}{k !}\left(\frac{\lambda \bar{\gamma}}{2(1+\bar{\gamma})}\right)^{k}\right]
\end{gathered}
$$

Considering the $i$ th cluster as an example, we have

$$
\begin{aligned}
& \Delta_{0, i}=P\left\{\lambda_{1, i}<E_{i, j}<\lambda_{2, i} \mid H_{0}\right\}=F\left(\lambda_{2, i}\right)-F\left(\lambda_{1, i}\right), \\
& \Delta_{1, i}=P\left\{\lambda_{1, i}<E_{i, j}<\lambda_{2, i} \mid H_{1}\right\}=G\left(\lambda_{2, i}\right)-G\left(\lambda_{1, i}\right),
\end{aligned}
$$

$\beta_{0, i}$ and $\beta_{1, i}$ represent the probabilities of events that all the $N_{i}$ SUs in the $i$ th cluster make no decisions under the hypothesis $H_{0}$ and $H_{1}$, respectively. Then

$$
\begin{aligned}
& \beta_{0, i}=\Delta_{0, i}^{N_{i}}=\left[F\left(\lambda_{2, i}\right)-F\left(\lambda_{1, i}\right)\right]^{N_{i}}, \\
& \beta_{1, i}=\Delta_{1, i}^{N_{i}}=\left[G\left(\lambda_{2, i}\right)-G\left(\lambda_{1, i}\right)\right]^{N_{i}} .
\end{aligned}
$$


If the number of the SUs making decisions in the $i$ th cluster under the hypothesis $H_{0}$ is denoted by $N_{0, i}$ and $N_{1, i}$ is the number of the SUs making decisions under the hypothesis $H_{1}$, the detection and false alarm probabilities given by the cluster head SU after receiving the decision results from the member SUs in the $i$ th cluster can be calculated as,

$$
\begin{aligned}
Q_{d, i} & =\left(1-\beta_{1, i}\right) \\
& \times\left\{1-\prod_{k=1}^{N_{1, i}}\left[G\left(\lambda_{2, i}\right)\left(1-P_{e, k, i}\right)+\left(1-G\left(\lambda_{2, i}\right)\right) P_{e, k, i}\right]\right\}, \\
Q_{f, i} & =\left(1-\beta_{0, i}\right) \\
& \times\left\{1-\prod_{k=1}^{N_{0, i}}\left[F\left(\lambda_{2, i}\right)\left(1-P_{e, k, i}\right)+\left(1-F\left(\lambda_{2, i}\right)\right) P_{e, k, i}\right]\right\},
\end{aligned}
$$

where $P_{e, k, i}$ represents the BEP of the inter-user channel of the SU $k$ to the head SU in the $i$ th cluster.

The head SUs of the $N$ clusters will send their decisions to the fusion centre and then the final decision will be made. Hence, the sensing performance of the whole system can be derived by replacing $P_{d, i}$ in (6) and $P_{f, i}$ in (7) with $Q_{d, i}$ and $Q_{f, i}$ obtained above, respectively.

\section{IV.SIMULATION RESULTS AND ANALYSIS}

In this section, the simulation results and advantage of the proposed cooperative spectrum sensing scheme are given. In the simulation, we assume that the detecting channels of the SUs follow the same Rayleigh fading distribution, the interuser channels and the reporting channels of the SUs follow different Rayleigh fading distribution to simulate the real environment.

The performance of the sensing bits of the proposed CSS is shown in Figure 4. It is shown that the proposed scheme is the same as that with one threshold when $\Delta_{0, i}=0(i=1,2)$ and the normalized number of the sensing bits $n_{\text {norm }}$, which is calculated as the ratio of the number of sensing bits when $\Delta_{0, i}$ does not equal 0 to that when $\Delta_{0, i}$ equals 0 , is the biggest. $n_{\text {norm }}$ becomes smaller gradually when $\Delta_{0, i}$ increases. For example, more than $30 \%$ reduction of the sensing bits is obtained when $Q_{f}$ is 0.01 . Meanwhile, $n_{\text {rat }}$, which denotes the ratio of the sensing bit number of the proposed scheme to that of the STBC based CSS, is the smallest compared with the other curves and it denotes that the sensing bits of the STBC based CSS scheme is the most.

The performance of the proposed CSS scheme is depicted in Figure 5 when $\Delta_{0, i}$ equals to $0.2(i=1,2)$. When the reporting channels experience fading, the performance of CSS degrades greatly, especially in the low bounder of $Q_{f}$. If the inter-user channels are considered, the clustering method only causes the enormous loss of sensing performance due to the impact of inter-user channel fading. Although the STBC based scheme can improve the sensing performance significantly, the bandwidth to transmit the sensing bits will be enormous according to Figure 4 . The proposed scheme could save a lot of bandwidth with some marginal loss of performance and the performance loss mainly occurs when $Q_{f}$ is larger than 0.5 , whereas $Q_{f}$ is usually demanded to be as small as possible.

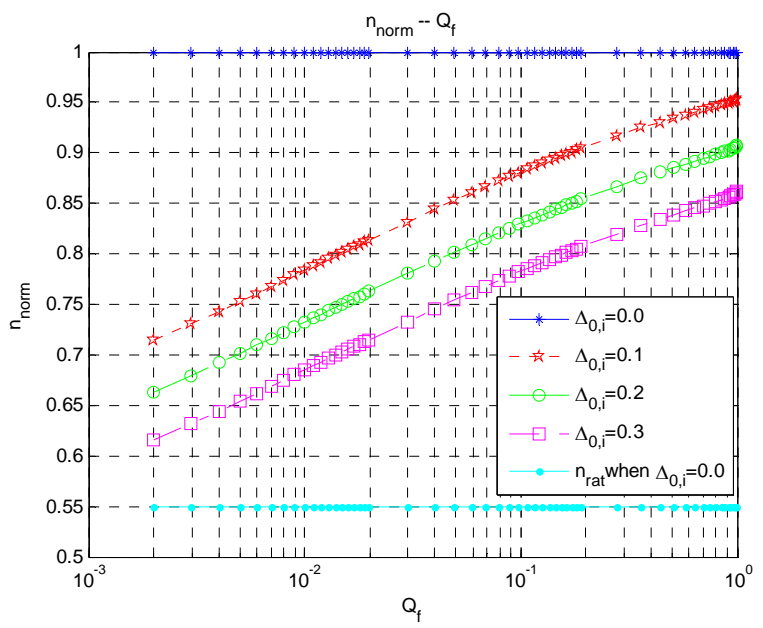

Figure 4. Performance of the sensing bit numbers of the proposed scheme compared with other cooprative spectrum sensing schemes

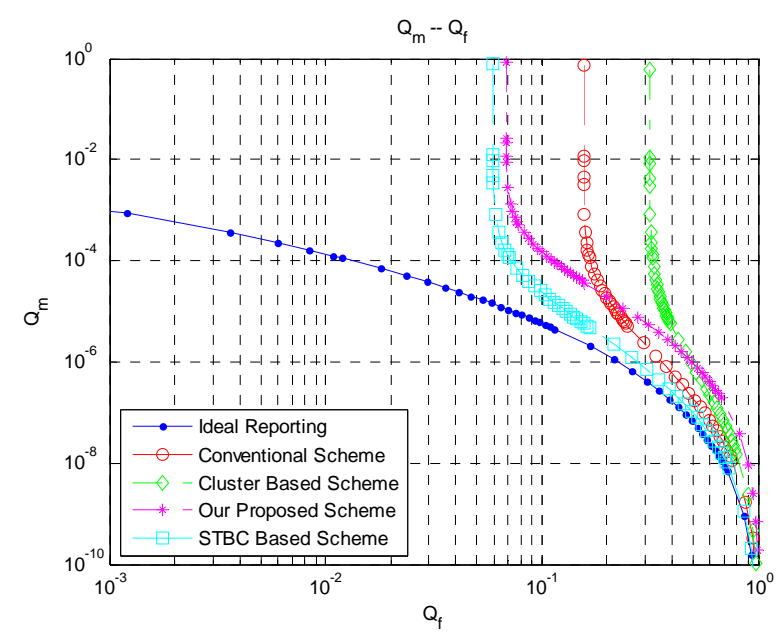

Figure 5. Performance of the proposed cooperative spectrum sensing

\section{Conclusions}

In this paper, a dynamical clustering cooperative spectrum sensing scheme with bandwidth constraints is proposed. Considering that all the channels in the CR system experience fading or shadowing and the bandwidth is limited, the proposed scheme can reduce the number of the transmission bits significantly and save the bandwidth in CR systems. Meanwhile, the system sensing performance loss is marginal. The proposed scheme is especially applicable to the situation where the bandwidth resource is scarce.

\section{ACKNOWLEDGEMENT}

This research is supported by National Science Foundation of China (No.61301147, No.61072040), Key Laboratory of Higher Education of Sichuan Province for Enterprise Informationlization and Internet of Things (2013WYJ02).

\section{REFERENCES}

[1] FCC, "Spectrum Policy Task Force: report of the Spectrum Efficiency Working Group”, Rep. ET Docket no. 02-135, Nov. 2002. 
[2] J. Mitola III and G. Q. maguire Jr., "Cognitive radio: making software radios more personal", IEEE Personal Commun. vol. 6, no. 4, pp. 1318, Aug. 1999.

[3] S. Haykin, "Cognitive radio: brain-empowered wireless communications", IEEE J. on Selec. Areas in Commun., vol. 23, no. 2, pp. 201-220, Feb.2005.

[4] W. Zhang and K. B. Letaief, "Cooperative spectrum sensing with transmit and relay diversity in cognitive radio networks", IEEE Trans. on Wireless Commun., vol. 7, no. 12, pp. 4761-4766, Dec. 2008.

[5] D. Cabric, S. M. Mishra and R. Broderson, "Implementation issues in spectrum sensing for cognitive radios", IEEE Proc. 38th Asilomar Conf. Signals, Systems and Computers, Pacific Grove, CA, vol. 1, pp. 772776, November 2004.

[6] A. Ghasemi and E. S. Sousa, "Collaborative spectrum sensing for opportunistic access in fading environments", IEEE Intl. Symp. on New Frontiers in Dynamic Spectrum Access Networks, pp. 131-136, 2005.

[7] C. Sun, W. Zhang and K. B. Letaief, "Cluster-based cooperative spectrum sensing in cognitive radio systems", IEEE Intl. Conf. on Commun., pp. 2511-2515, Jun. 2007.

[8] Y. Wang, C. Feng, Z. Zeng and C. Guo, "A robust and energy efficien cooperative spectrum sensing scheme in cognitive radio networks", 11th Intl. Conf. on Advance Commun. Tech., vol. 1, pp. 640-645, 2009.

[9] C. Sun, W. Zhang, and K. B. Letaief, "Cooperative spectrum sensing for cognitive radios under bandwidth constraints", IEEE Wireless Commun. and Networking Conf., pp. 1-5, Mar. 2007.

[10] Z. Bai, L. Wang, H. Zhang et al, "Cluster-based cooperative spectrum sensing for cognitive radio under bandwidth constraints", IEEE Intl. Conf. on Commun. Systems, pp. 569-573, Nov. 2010.

[11] H. Urkowitz, "Energy detection of unknown deterministic signals", Proceedings of IEEE, vol. 55, pp. 523-531, Apr. 1967.

[12] A. H. Nuttall, "Some integrals involving the QM function", IEEE Trans. on Inf. Theory, vol. 21, no. 1, pp. 95-96, Jan. 1975.

[13] I. S. Gradshteyn and I. M. Ryzhik, Table of Integrals, Series, and Products. San Diego, CA: Academic Press, 6th ed., 2000.

F. F. Digham, M. S. Alouini, and M. K. Simon, "On the energy detection of unknown signals over fading channels", IEEE Intl. Conf. on Commun., vol. 5, pp. 3575-3579, May 2003.

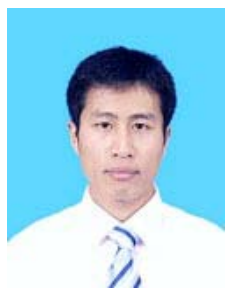

Bing Wang was born in Shandong province in 1986 $\mathrm{He}$ received his BS degree from Xi'an Jiaotong University, Xi'an, China in 2010 and became a MS student majored in Communications and Information Systems in School of Information Science and Engineering, Shandong University, Jinan, China from 2011. His major research field is spectrum sensing in cognitive radio systems.

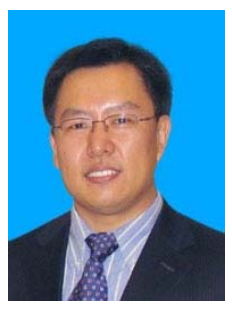

Zhiquan Bai received his BS degree from Inner Mongolia University, Huhehot, China, in 2000, MS degree from Shandong University, Jinan, China, in 2003, and $\mathrm{PhD}$ degree with honor from INHA University under the Grant of Korean Government IT Scholarship, Korea, in 2007. From 2007 to 2008, he was a post-doctor in UWB Wireless Communications Research Center, INHA University, Korea. After that, he has been an associate professor in the School of Information Science and Engineering, Shandong University, China. His current research fields include cooperative communication and MIMO system, cognitive radio, ultra wideband technologies and advanced channel coding and modulation.

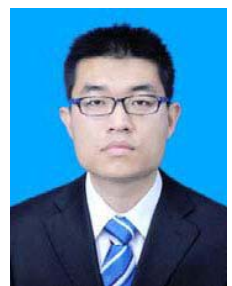

Yinlong Xu was born in Henan province in 1988. He joined the wide-band wireless communication research lab of Shandong University, Jinan, China in 2011. His research interests include cooperative communication.

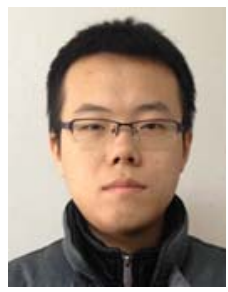

Peihao Dong was born in Shandong province in 1990. He received his BS degree from Shandong University, Weihai, China, in 2012. Now he is a MS student in School of Information Science and Engineering, Shandong University, Jinan, China. His research interests include cooperative communication.

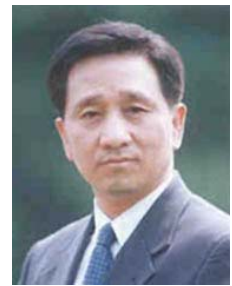

Kyungsup Kwak received his BS degree from Inha University, Incheon, Korea, in 1977, MS degree from the University of Southern California in 1981, and PhD degree from the University of California at San Diego in 1988. From 1988 to 1989 , he was a member of Hughes Network Systems, San Diego, California. From 1989 to 1990 , he was with the IBM Network Analysis Center, North Carolina. Since then, he has been with the School of Information and Communication, Inha University, Korea, as a professor. He is the director of UWB Wireless Communications Research Center in Inha University and Inha Fellow Professor (IFP). His research interests include multiple access communication systems, mobile communication systems, UWB radio systems, and ad hoc networks, as well as high-performance wireless Internet. 